OPEN ACCESS

Edited by: Richard Porter,

University of Otago, New Zealand

Reviewed by:

Carlo Lai,

Sapienza University of Rome, Italy

Frederick Sundram The University of Auckland,

New Zealand

${ }^{*}$ Correspondence:

Pei Sun

peisun@tsinghua.edu.cn

Shuquan Chen

sc4173@tc.columbia.edu

Specialty section:

This article was submitted to Mood and Anxiety Disorders,

a section of the journal

Frontiers in Psychiatry

Received: 20 August 2020 Accepted: 29 January 2021

Published: 19 February 2021

Citation:

Han X, Chen S, Bi K, Yang Z and Sun $P$ (2021) Depression Following COVID-19 Lockdown in Severely, Moderately, and Mildly Impacted

Areas in China.

Front. Psychiatry 12:596872. doi: 10.3389/fpsyt.2021.596872

\section{Depression Following COVID-19 Lockdown in Severely, Moderately, and Mildly Impacted Areas in China}

\author{
Xuerui Han ${ }^{1}$, Shuquan Chen ${ }^{1 *}$, Kaiwen $\mathrm{Bi}^{2}$, Zeyun $\mathrm{Yang}^{3}$ and Pei Sun ${ }^{3 *}$ \\ ${ }^{1}$ Department of Clinical and Counseling Psychology, Teachers College, Columbia University, New York, NY, United States, \\ ${ }^{2}$ Department of Psychology, Columbia University, New York, NY, United States, ${ }^{3}$ Department of Psychology, School of Social \\ Science, Tsinghua University, Beijing, China
}

Background: The Coronavirus Disease 2019 (COVID-19) pandemic has led to overwhelming levels of distress as it spread rapidly from Wuhan, Hubei province to other regions in China. To contain the transmission of COVID-19, China has executed strict lockdown and quarantine policies, particularly in provinces with the highest severity (i.e., Hubei). Although the challenges faced by individuals across provinces may share some similarities, it remains unknown as to whether and how the severity of COVID-19 is related to elevation in depression.

Methods: The present study compared depression among individuals who lived in mildly, moderately, and severely impacted provinces in China following the lockdown ( $N$ $=1,200$ ) to norm data obtained from a representative sample within the same provinces in 2016 ( $N=950$ ), and examined demographic correlates of depression in 2020.

Results: Residents in 2020, particularly those living in more heavily impacted provinces, reported increased levels of depression than the 2016 sample. Subsequent analyses of sub-dimensions of depression replicated the findings for depressed mood but not for positive affect, as the latter only declined among residents in the most severely impacted area. Increased depressed mood was associated with female, younger age, fewer years of education, and being furloughed from work, whereas reduced positive affect was associated with younger age and fewer years of education only.

Conclusions: This study underscored the impact of COVID-19 on depression and suggested individual characteristics that may warrant attention.

Keywords: exposure, stress, lockdown, COVID-19, depression

\section{INTRODUCTION}

In December 2019, an unknown and infectious disease broke out in Wuhan, China, which was declared a pandemic by the World Health Organization (1) and officially referred to as the Coronavirus Disease 2019 (COVID-19) on February 11th, 2020. Since then, it has taken a tremendous toll on individuals, families, and communities, infecting and claiming millions of lives worldwide (2). In addition to adapting to a new reality dominated by fear of viral contagion as well as social isolation due to lockdown and quarantine, individuals have experienced devastating losses in multiple domains of life. The long-term effects may extend further than the duration of 
the lockdowns. As individuals start to seek reemployment and a return to normal life, depressive symptoms may emerge as feelings of sadness and loss grow beyond fear and anxiety, when they realize that normality continues to remain elusive. The present study aims to compare depressive symptoms among Chinese residents in provinces that were severely, moderately, mildly hit by the pandemic in early May of 2020 with pre-pandemic norm and identify demographic correlates of depression after the lockdown.

\section{Elevated Psychological Distress Amidst the Outbreak of COVID-19}

COVID-19 is particularly disruptive as it imposes widespread and severe restrictions without a certain end date, presents a complex combination of stressful life events, and blocks access to protective factors (3).

Fear of contracting the virus is probably one unique stressor that the COVID-19 has imposed on individuals. Over onethird of respondents expressed increased concern and excessive anxiety about viral infection, even when the risk was estimated to be low in early 2020 (4). Loss due to COVID-19 can range in severity and duration, be direct (i.e., infection) or indirect [i.e., child mortality in low-income countries; Roberton et al. (5)], present-oriented (i.e., unemployment), or future-oriented (i.e., uncertainty of academic progression) and on the individual (i.e., increased psychological distress), or the collective (i.e., an overstretched medical system) level, resulting in varying elevation in psychological distress. Another ramification of COVID-19 is an increase in the feelings of disgust toward outgroups that are believed to pose an elevated risk of infection (6). Disease avoidance arises from people's evolutionary tendency to maintain health (7), but it unfortunately contributes to prejudice against national subgroups (i.e., the residents of Hubei Province, China). Hubei residents encountered increasing social exclusion and stigmatization in forms of in-person verbal assault, destruction of property, being denied employment opportunities or access to public facilities and a general violation of fairness (8). Given that the adverse impact of discrimination on people's mental health has been well-documented (9), it is possible that levels of depression in Hubei residents might be further aggravated by such experiences.

\section{Depression Following the Lockdown}

COVID-19 led to unprecedented policies of quarantine in an attempt to contain the pandemic, starting with Wuhan in Hubei Province. Enforced by government and community officials, stringent lockdown measures prohibited residents from leaving the city, restricted each household to send one person to purchase groceries twice a week, and banned the private use of cars (10). To further limit group activities, the local government also took steps to reward individuals who reported neighbors breaking social distancing rules (10). Inevitably, mandatory quarantine generated common challenges such as working from home while balancing childcare, experiencing wage loss, and lacking food supplies, and clashed with the fundamental human need for connection and belonging (11). Forced social isolation reduced social and physical contacts with others, thereby generating elevated depressed mood, emotional disturbance, boredom, frustration, and blocking access to effective coping strategies such as seeking social support $(12,13)$.

\section{Stress Exposure: Severely, Moderately, and Mildly Impacted Areas}

In the current literature, a number of studies have investigated the association between combat exposure and the prevalence of PTSD and mood disorders, provided that exposure to trauma is positively related to the severity of symptoms (14-16). Other studies have followed to suggest that the magnitude of exposure to a variety of adverse events, including natural disasters (17), childhood maltreatment (18), and racism (9) is associated with subsequent depressive symptoms and overall maladjustment (17). Studies on different epidemics, including COVID-19, the severe acute respiratory syndrome (SARS), and Middle East respiratory syndrome (MERS), reached same conclusions that the level of disease exposure was a substantial risk factor for developing psychological problems (19). Specifically, health care workers or employees in high-exposure-risk locations (i.e., Wuhan vs. other cities in Hubei province vs. outside Hubei province) were significantly more likely to exhibit symptoms of depression, anxiety, distress, insomnia (20), and PTSD symptoms (21). Therefore, it is reasonable to suspect that different patterns of mental health issues existed across provinces that were exposed to COVID-19 to different degrees.

One way to determine the severity of exposure for each province is calculating the infection rate. Specifically, the provincial number of COVID-19 cases was divided by the total number of regular residents (in millions) for each province, using the government census data. The infection rate, ranging from 2.99 to $1,151.41$, was utilized as an index to compare severity and categorize all regions into mildly impacted (MiA, i.e., 2.999.03), moderately impacted (MoA, i.e., 12.24-27.53), and severely impacted areas (SeA, 1,151.41). By May 10th, 2020, Hubei province is considered the highest in severity with an infection index of 1,151.41 to represent SeA. For MoA, Guangdong and Zhejiang provinces were chosen, with 22.10 and 14.00 per million residents contracting the virus. Shanxi and Sichuan represented MiA, since both provinces had lower infection indexes of 5.33 and 6.73, respectively. Hubei, Guangdong, Zhejiang, Sichuan, and Shanxi provinces are geographically proximate.

\section{Demographics Correlates of Depression Following the Lockdown}

Recent research has identified potential correlates such as younger age, being single, fewer years of education, female gender, student status, pre-existing physical symptoms, and poor perceived general health (22-24). In an attempt to replicate previous findings and generate novel explanations, we included not only gender, age, years of education attained, and marital status, but also annual income and changes in work or wage resulting from disruptions caused by COVID19 into the analysis. To our knowledge, these two factors were rarely discussed in combination. Early works showed that unemployment and economic insecurity had detrimental effects 
on one's self-rated health and psychological health both shortterm and long-term (25-27). Following the Great Economic Regression from 2007 to 2009, recession-related stressors such as increased debt, reduced budget, unemployment, and inability to pay rent, were associated with higher odds of developing depression, generalized anxiety, panic, substance use even years later (28). Similarly, Wilson et al. (29) have found that increased job insecurity and financial concerns were differentially associated with heightened depressive and anxious symptoms. Brooks et al. (12) have proposed that individuals with lower annual income prior to the pandemic might be more affected by financial uncertainties and require additional support than those with higher income. Taking both income level and change in employment status into account, we intended to investigate which factor was more strongly related to increased depression in face of COVID-19. The majority of research on COVID-19 has used univariate analyses to explore these relationships, whereas our study conducted multivariate analyses with a forward stepwise procedure (30), which could provide information on the significance of the relationships and the size of the effects as well as the structure and the interaction effect of multiple covariates while adjusting for potential confounding factors (31).

\section{The Current Investigation}

The current investigation aims to compare depression along with its two subdimensions, measured by the Center for Epidemiological Studies - Depression (CES-D) scale among residents living in mildly, moderately, and severely impacted provinces in 2020 and norm data in 2016. In addition, we investigated how potential demographic factors relate to depression. Taken together, we proposed the following hypotheses: (1) Residents in 2020 would exhibit greater levels of depression than residents in 2016. (2) Among residents surveyed in 2020, those living in SeA would exhibit the highest levels of depression, followed by those living in MoA, followed by those living in MiA. (3) Following the lockdown, those who were female, furloughed, or achieved lower levels of education and income would exhibit greater levels of depression than their counterparts. The other demographic factors examined were exploratory in nature, including age (i.e., while age was often perceived to be negatively correlated with depression, the elderly might have suffered the most during the pandemic) and marital status (i.e., although this factor was often discussed, research results were inconsistent).

\section{METHODS}

\section{Participants and Data}

To capture levels of depression prior to COVID-19, depression norm data collected in 2016 from the China Family Panel Studies [CFPS; (32)], a nationally representative survey of Chinese communities, families, and individuals, was obtained. CFPS is conducted by the Institute of Social Science Survey (32) of Peking University, China, attempting to provide a comprehensive overview of the citizen's health, mental wellbeing, educational attainment, family income, parental practices, social relationships, and others. CFPS collects data every 2 years, and the most recent data set was sampled in 2018. Nonetheless, it did not measure the 20-item CES-D scale and could not be used. Instead, the openly available 2016 data set included the CES-D 20 -item scale and served as the baseline depression norm, which is comparable to those of two other Chinese studies $(33,34)$. Eligibility criteria included age between 18 and 65, responding to all 20 items on the CES-D scale, and living in Hubei, Guangdong, Zhejiang, Sichuan, or Shanxi at the time when the survey was taken. Data were collected in person, through the phone, or using the internet. Participants who did not comply with data collection (e.g., invariance of response or non-compliance) were excluded.

All participants in the 2020 sample were recruited simultaneously from May 10 to 20, 2020, adopting the same eligibility criteria. The study was launched using the Questionnaire Star, a Chinese survey platform that facilitates high-quality data collection. A link to the survey was disseminated via popular social media platforms such as Wechat, Weibo, and Zhihu. Participation was voluntary and anonymous. Respondents were debriefed about the nature and aim of the study and gave informed consent. This study was approved by the Institutional Review Broad (IRB) at the Department of Psychology, XXX (Masked for blind review) University.

\section{Measures}

The survey consisted of demographic information and depression scores. Participants were asked to report their age, gender, education level, marital status, their annual individual income prior to COVID-19 and changes in their employment and income status (i.e., "Decreased income," "No change in income," "Increased income," "Being furloughed," "No employment") at the time of the survey. Gender (female $=0$, male $=1$ ), marital status (single $=1$ ), income change (furlough, decreased income $=1$ ) were dummy coded. The reduced wage was coded together with being furloughed to capture the negative effects of COVID-19 on individuals' or familial financial capacities. In the 2020 data, provinces were coded according to the severity of exposure, with Hubei Province being 3, Guangdong and Zhejiang being 2, and Sichuan and Shanxi being 1 .

Depression was measured with the 20-item CES-D scale (35), which captured an individual's level of depression and the frequency of thoughts or behaviors during the past week and used a three-point scale from 0 ( $<1$ day a week) to 3 (57 days a week). The total score ranged from 0 to 60 , with a higher rating indicating a more severe presentation of depression. Although Radloff's (35) original work supported a four-factor model of CES-D (i.e., depressed affect, positive affect, somatic and retarded activity, and interpersonal factor), the current investigation adopted a two-factor model to avoid potential overextraction (36). The two factors were relevant to the wording of the items as four of them were positively valenced and the remaining negatively valenced (37). Factor positive emotion included item $4,8,12$, and 16 , and the remaining items summed to reflect the second factor depressed mood. 


\section{Analytic Plan}

All statistical analyses were performed in $\mathrm{R}$ (38) via glm in base $\mathrm{R}$, and pequod, huge, car, tidyverse, lm.beta, lme4, WRS packages. To investigate the significance of differences in scores obtained from CES-D among the four groups (2016 and Mildly, Moderately, and Severely Impacted Areas in 2020) categorized by the levels of severity at which an area was hit by COVID19, analysis of variance (ANOVA) or its variant would be applied after the assumptions of equal variance and normality were tested.

To explore the relationship between demographic factors and depression, depressed mood, and positive affect in 2020, three hierarchical multiple regression models were built. Standardized coefficients $(\beta)$ were provided for regression analyses. Simple slope analysis $(39,40)$ was conducted on interaction effects to reveal the nature of significant interactions and detect relations between predictors and outcomes at different levels of the moderator with increased sensitivity (41). Compared to the test of interaction effect in a regression model, a test of simple slopes has increased power regardless of the interaction term's significance and decreased likelihood of Type II error, while maintaining an equivalent level of Type I error (41). Severity was set as the moderator for all analysis with each slope assessed at "low" (1 $S D$ below the mean) and "high" (1 SD above the mean) levels of severity.

\section{RESULTS}

From the complete CFPS 2016 data set, a total of 950 respondents at their middle age $(M=43.33, \mathrm{SD}=13.57)$ were included for the calculation of norm and final analysis. The majority of respondents were women (54.8\%), married, divorced, or widowed (87.1\%), and attended high school education or less $(80.4 \%)$.

For the 2020 sample, 1,200 participants at their middle age ( $M$ $=31.18, S D=11.59$ ) were eligible for final analyses. The sample size was moderate across Hubei $(N=300$, Age: $M=29.26, S D$ =9.99), Guangdong $(N=199$, Age: $M=28.82, S D=8.40)$, Zhejiang $(N=201$, Age: $M=28.37, S D=10.24)$, from Sichuan $(N=249$, Age: $M=27.51, S D=8.89)$, and Shanxi $(N=251$, Age: $M=41.26, S D=13.27)$. Among them, a majority were women $(64.1 \%)$, single or never married (53.1\%), had a master's degree or less (86.2\%), an annual income of 50,000 CNY or less (48.2\%), and did not report changes in their work or income status (23.6\%).

\section{Change in Depression From 2016 to 2020}

At the traditional CES-D cutoff value of 16 (35), which proposes that people who score equal to or above 16 are at risk for clinical depression, the relation between these variables was significant, $X^{2}(1, N=2,150)=112.87, p<0.001$, and $29,44,49$, and $54 \%$ residents met this criterion of depression in 2016, MiA, MoA, and $\mathrm{SeA}$, respectively. Following the recommendations of Vilagut and colleagues (42) who proposed 20 as a better cutoff point, the relation was still significant, $X^{2}(1, N=2,150)=97.95, p<0.001$, and $16,32,35$, and $40 \%$ residents met this criterion of depression in 2016, MiA, MoA, and SeA, respectively.
As the data collected violated the assumptions (i.e., assumption of normality and equal variances) of traditional ANOVA, robust ANOVAs and robust post-hoc tests based on bootstrapping and trimmed means were chosen, as they could yield more accurate results when assumptions are not met (43). In total, three one-way robust ANOVAs were specified to evaluate the differences among four groups on total depression score, depressed mood, and positive emotion (Table 1 and Figure 1).

A robust ANOVA examining possible differences among the four groups was significant, $F t=45.15, p<0.001$, indicating that some groups reported elevated levels of depression than others. The results of robust post-hoc tests were all significant except for the comparisons between the scores of residents who lived in MoA and those of residents who lived in SeA, $p=0.10$ and between MiA and MoA, $p=0.14$. Notably, the robust post-hoc comparisons revealed that the residents in 2016 were significantly less depressed than those of residents living MiA, $p<0.001$, Hedges' $g=0.45$, MoA, $p<0.001$, Hedges' $g=0.58$, and SeA, $p<0.001$, Hedges' $g=0.75$, in 2020 .

The second robust ANOVA revealed significant differences across groups in depressed mood, $F t=61.65, p<0.001$. The results of robust post-hoc tests were almost always consistent with the previous post-hoc test on total depression: all results were significant except for the comparison between MoA and SeA, $p$ $=0.16$. Residents assessed in 2016 exhibited significantly lower levels of depressed mood than those in MiA, $p<0.001$, Hedges' $g=0.56$, MoA, $p<0.001$, Hedges' $g=0.66$, and SeA, $p<0.001$, Hedges' $g=0.80$, in 2020 .

A final robust ANOVA on positive emotion also yielded a significant result, $F t=7.39, p<0.001$, indicating that some groups experienced lower positive mood. The results of posthoc tests, however, revealed a different pattern. Residents in SeA exhibited reduced positive mood than all other groups, p.s. $<$ 0.01 , Hedges' g.s. $<-0.21$. The level of positive emotions of residents in MoA and MiA in 2020 were not statistically different from those of residents assessed in 2016.

\section{Symptom Variability Within 2020}

For the total score of depression (Table 2), in step 1, age was found negatively associated with depression, $\beta=-0.21, t_{(1197)}$ $=-7.36, p<0.001$. In step 2, main effects of educational attainment, marital status, income level, and income change due to COVID-19 were included. As expected, a negative relationship between education and depression was found, $\beta=$ $-0.09, t_{(1178)}=-3.14, p=0.002$. Moreover, the experience of being furloughed was significantly associated with increased depression, $\beta=0.11, t_{(1178)}=3.62, p<0.001$. Severity of COVID-19 was included to the model in step 3 , but there was no evidence supporting the link between provincial severity of COVID and depression, $\beta=0.04, t_{(1177)}=1.53, p=0.13$. In step 4 , six two-way interaction terms, between gender and severity, age and severity, education and severity, being single and severity, income and severity, and being furloughed and severity were included. Among them, only the one between gender and severity was significant, $\beta=-0.19, t_{(1171)}=-2.53, p=0.01$. Simple slope analyses were performed. Compared to men, women were more 
TABLE 1 | Depression in Residents Assessed in 2016, MiA, MoA, and SeA and Symptom Comparisons.

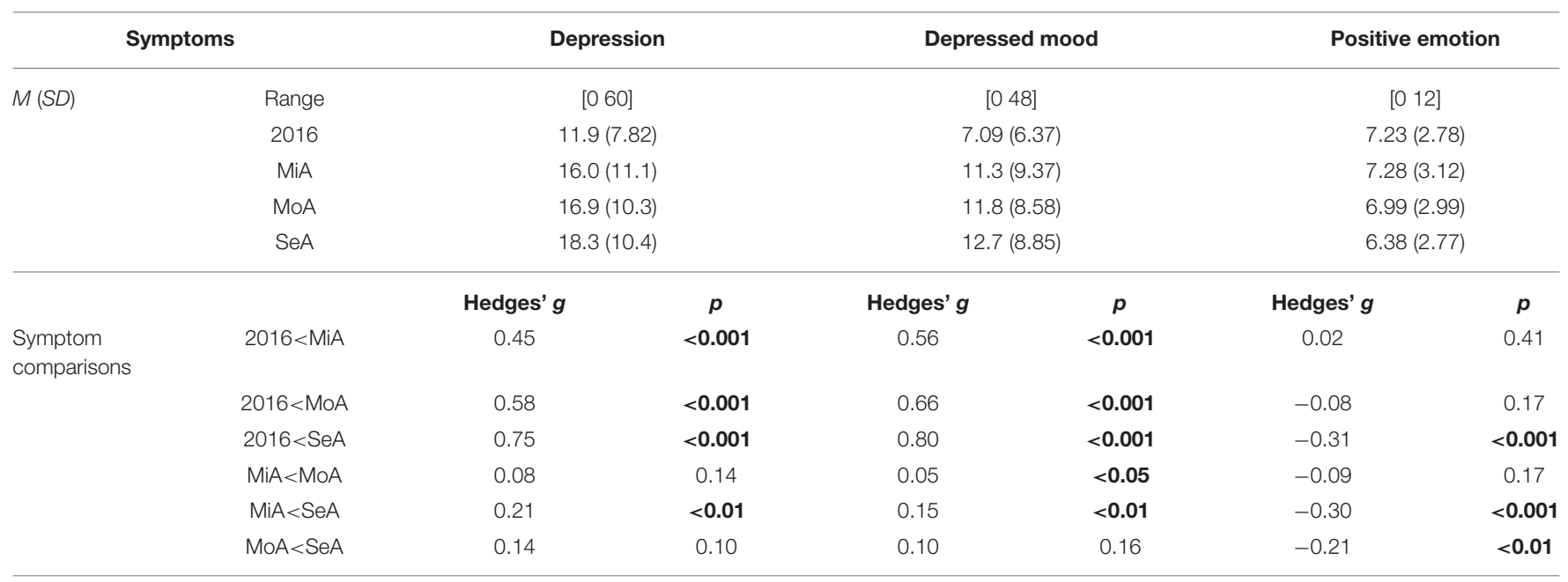

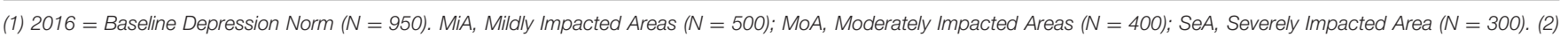

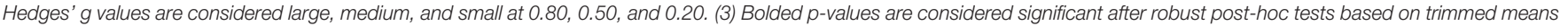
and bootstrapping with false discovery rate set to be 0.05 .

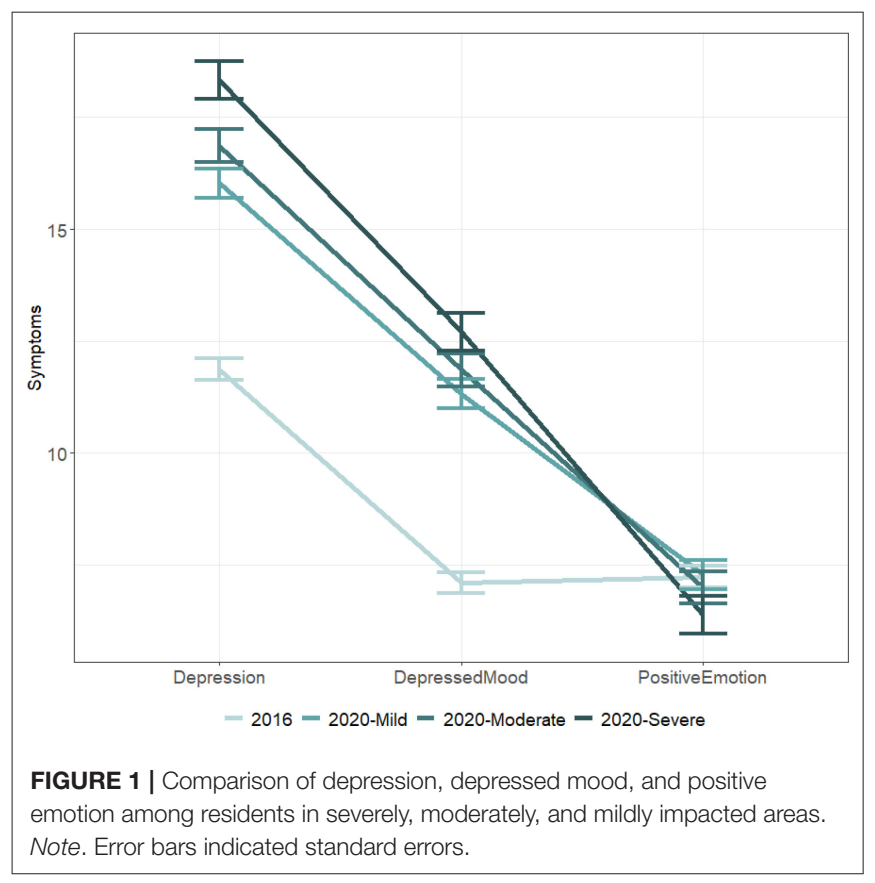

likely to experience depression when the severity was high, $B=$ $-2.67, p=0.006$, but not when the severity was low, $B=-0.09$, $p=0.92$. The marginally significant interaction effect between education and severity, $\beta=0.57, t_{(1171)}=1.95, p=0.05$, was also tested. The slope was significantly different than zero at a low level of severity, $B=-0.90, t_{(1171)}=-3.53, p<0.001$, yet insignificant at a high level of severity, $B=-0.15, t_{(1171)}$ $=-0.60, p=0.55$, suggesting that only in provinces that were less severely impacted, as years of education increased, the total CES-D score decreased.

Similar findings emerged for depressed mood (Table 2). In step 1, both gender, $\beta=-0.06, t_{(1197)}=-2.12, p=0.03$, and age, $\beta=-0.22, t_{(1197)}=-7.95, p<0.001$, were significantly related to changes in depressed mood. In step 2, educational level, $\beta=-0.07, t_{(1178)}=-2.39, p=0.02$, was negatively associated with depressed mood, while being furloughed, $\beta=$ $0.11, t_{(1178)}=3.76, p<0.001$, was positively associated with depressed mood. In step 3 , severity was not related to changes in depressed mood, $\beta=0.01, t_{(1177)}=0.39, p=0.70$. In step 4 , only the interaction between gender and severity demonstrated a meaningful relationship to depressed mood, $\beta=-0.19, t_{(1171)}$ $=-2.57, p=0.01$, whereas the interaction between education level and severity was marginally significant, $\beta=0.51, t_{(1171)}=$ $1.72, p=0.09$. Therefore, simple slope analyses were performed to reveal the nature of interaction. Comparable to depression, females who lived in more severely impacted regions experienced increased depressed mood compared to males, $B=-2.73$, $t_{(1171)}=-3.37, p<0.001$, but not when they lived in less severely impacted areas, $B=-0.45, t_{(1171)}=-0.60, p=0.55$. Education, again, was a significant predictor of depressed mood only when severity was low, $B=-0.60, t_{(1171)}=-2.77, p=$ 0.006 , but not when severity was high, $B=0.008, t_{(1171)}=0.04$, $p=0.97$.

Age was positively associated with positive emotions (Table 2), $\beta=0.07, t_{(1197)}=2.50, p=0.01$. In step 2, there was strong evidence that more years of education were associated with greater positive emotion, $\beta=0.12, t_{(1178)}=3.99, p<0.001$. Neither income nor being furloughed was significant. In step 3 , results demonstrated that, as severity increased, positive emotion decreased, $\beta=-0.12, t_{(1177)}=-4.23, p<0.001$. In the last step, the final model supported the significance of education, single status, p.s. $<0.05$, and the marginal significance of the interaction effect between educational attainment and severity, $\beta=-0.52, t_{(1171)}=-1.76, p=0.08$. Well-educated individuals were less susceptible to a drop in positive emotion in both less and more severely affected regions, though the slope at low severity, $B=0.30, t_{(1171)}=4.30, p<0.001$, was steeper and 
TABLE 2 | Standardized regression coefficients and accounted variances for hierarchical multiple regression.

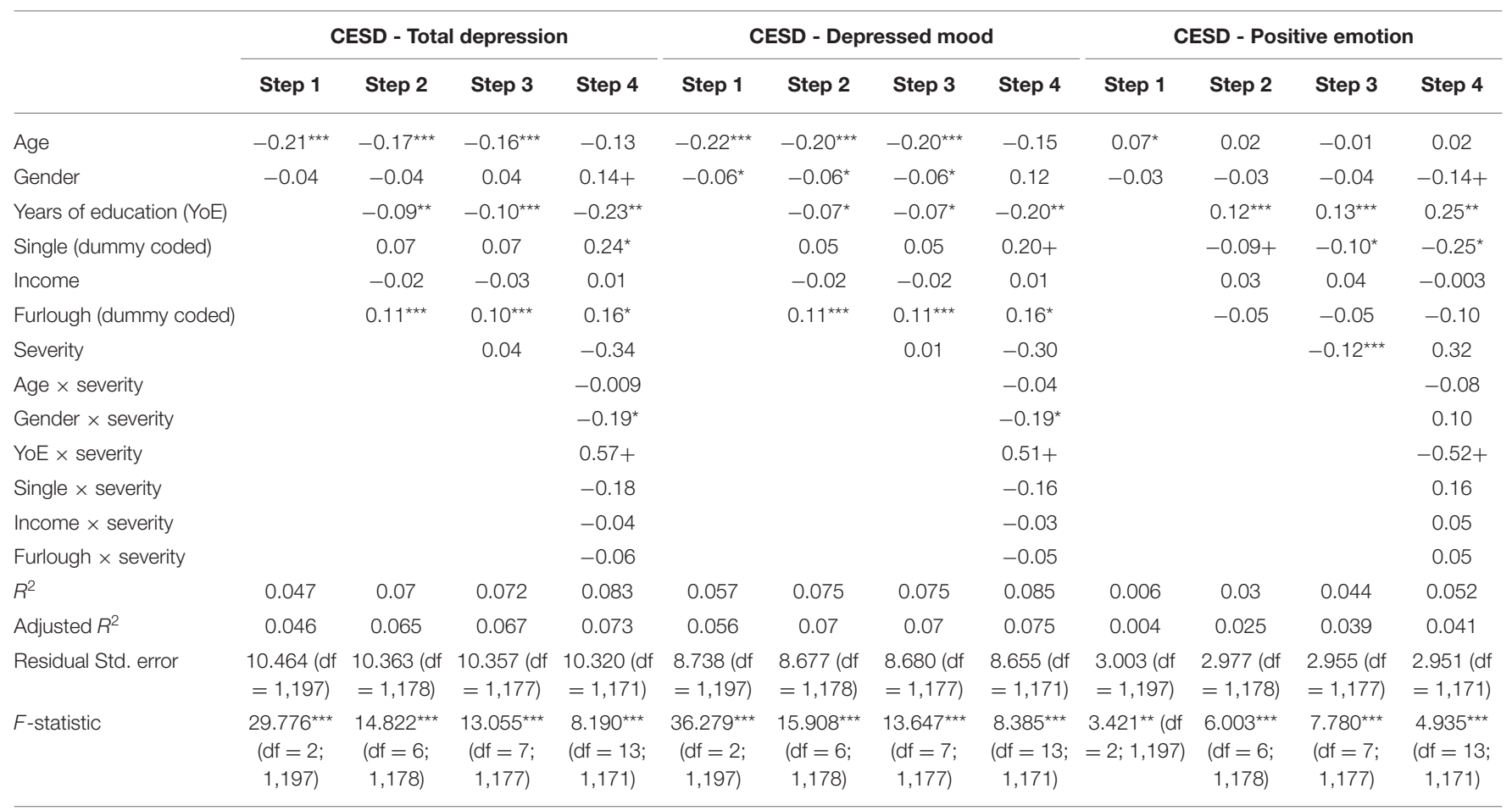

Note:,$+ p<0.10 ;{ }^{*}, p<0.05 ;{ }^{* *}, p<0.01 ;{ }^{* * *}, p<0.001$.

signified a greater power than the slope at high severity, $B=$ $0.16, t_{(1171)}=2.26, p=0.02$.

\section{DISCUSSION}

Amid the global outbreak of COVID-19, individuals may experience increased distress.

In accordance with the first hypothesis, participants recruited in the early May of 2020 reported greater severity of depression as compared with a pre-pandemic norm established using representative samples in 2016. It should be noted that there were age differences and that the simple manipulation of limiting the sample to an age range (i.e., 18-65) could not guarantee the equivalence of central measures between these group. Within in the 2020 sample, elevation of symptoms differed significantly from MiA, to SeA, determined by the provincial infection rates. Our results have provided confirming evidence for the second hypothesis as well as previous studies suggesting that increased exposure to COVID-19 through location, media, or infected cases predicted mental health problems (44). High levels of depression may also reflect comorbid anxiety, posttraumatic stress disorder, sleep disorder, suicidal ideation, domestic violence, substance use disorder as tested in other studies, and potentiate longterm consequences like cognitive impairments, psychosomatic symptoms, and behavioral changes (45).

Depressed mood, like the total level of depression, was higher in 2020 and positively associated with the severity of COVID19. Findings from the robust ANOVA confirmed that people facing COVID-19 experienced a significantly higher degree of depressed mood, which was intensified by increased exposure to COVID-19, as symptoms were significantly lower in MiA than MoA and SeA.

A separate pattern emerged for positive emotion, which did not differ among the baseline norm, MiA, and MoA. Only residents of SeA had significant impairments in positive emotion when compared to the other three groups. Perceived discrimination may partly account for this observation, since according media only residents of Hubei (i.e., SeA) reported various forms of prejudice. Previous studies have established a relationship between perceived discrimination and alterations in affect, especially in stressful situations like the current one (46). One way to explain the distinct pattern emerged for positive emotion could be that the pandemic primarily exerted its negative impact through aggravating depressed mood without necessarily reducing positive affect.

\section{Demographic Correlates}

\section{Age and Marital Status}

In the 2020 sample, people who were younger and single were affected more heavily by COVID-19 and more likely to have depression. People who were older reported lower levels of depressed mood and higher levels of positive emotions. Marital status was not associated with depressed mood or positive mood in our study.

In regard to age, Qiu et al. (23) showed that individuals between the ages of 18 and 30 or above 60 had the highest level of distress, recognizing that young people were more susceptible to stress-inducing information on social media and 
individuals older than 60 were more likely to feel threatened by the high mortality rate of COVID-19 among elderly. Our findings have partly confirmed their results, as younger individuals in the current sample were more likely to be depressed, have depressed mood, or to experience decreased positive emotions. First, young and single individuals had easy access to social media platforms, were more motivated to seek health, informational, and social support online, and more likely to get overwhelmed by the combination of accurate and faulty information (47). Second, they were more likely to quarantine alone and fared the worst with greater levels of future uncertainty in terms of academic and career progression (24). Third, higher levels of loneliness, financial distress, sleep problems, perceived stress and anxiety, and lower resilient coping were found among the younger population, compared to the older population (48). Furthermore, prior studies regarding SARS in 2003 have underscored the interplay between infection control measures, like wearing a mask and confinement within the home, to reinforce a sense of isolation (49) and subjective loneliness (50), which typically differed from an active lifestyle brimmed with vigor and gregariousness. A dramatic and enforced change in the frequency of social interactions and lonely feelings exerted detrimental effects on health, including impaired functionality, perceived decline in life quality and self-rated health (50).

\section{Gender}

Inconsistent with parts of the third hypothesis, gender was marginally significantly associated with depressed mood, but not with depression or positive emotion. Female participants reported higher levels of depressed mood as compared to their male counterparts. Our findings were partly consistent with relevant studies which proposed that females had a greater risk for depression, anxiety, and stress across nations during the pandemic, although these studies did not specify any subdimensions of depression (51-53). Gender differences in depression have been long established, looking at this issue through biological, psychological and social lenses (54). Under the unique circumstances of a global pandemic, the quarantine order might have led to forced and unwanted proximity with others, exposing women to escalated relationship difficulties or interpersonal problems. In extreme cases, rates of domestic violence grew as tensions built at home and victims were involuntarily confined with their abusers (55). It also pushed women to accept an overload of roles within the household and outside of it while adjusting to additional responsibilities (54).

\section{Educational Attainment}

Unlike other research in which the effect of education has been inconsistent (22-24), we validated parts of the third hypothesis concerning the effect of education and found that more years of education were promising in decreasing depression, depressed mood, and increasing positive emotions. Education could protect against both persistent sadness and anhedonia or diminished positive affect. Education may prove useful when dealing with stressful life events, thereby decreasing the likelihood of lifetime depression (56-58). Another potential explanation could be that individuals with lower levels of education were often subject to a furlough or a permanent layoff at times of an economic recession (59).

\section{Employment and Income}

The individual income level prior to the pandemic was not associated with depression, depressed mood, or positive emotions. Results contradicted our hypothesis and previous research (12). For example, Ettman et al. (59) saw a higher prevalence of financial stressors and probable depression in people with fewer assets, defined by household income, savings, house ownership, education, and being married. Another study reported that families with lower income levels had elevated symptoms of depression, anxiety, insomnia, and acute stress across various regions in China (60). On the contrary, high monthly income was found to be a risk factor for depression, anxiety, and sleep disorder by Wenning et al. (61). Discrepancies in conclusions may suggest a complex picture of the relationship between income, financial assets, and risk of depression, possibly mediated by geographical location, parental financial support, and culture-specific spending and saving practices. Geographical location determines a city's level of urbanization, the proportion of migrant workers, and living or rental housing unaffordability issues (62). Even though household income was generally higher in the first-tier cities, individuals, especially migrant workers, faced the reality of high rent stress (i.e., with a rent-to-income ratio going up to $50 \%$ in some cities), spatial inequality, and uncertainty due to short-term lease contracts (63). Parental financial support might be a potential confounder in our study, considering that we measured individual income per year without taking parental contribution into account. In light of the traditional values that held interdependence in high regard, Chinese parents are typically more determined to support their children financially until the children bear the role of a supporter (64). Moreover, with a high national savings rate of 59\% in 2012 (65), the Chinese samples might have more savings in immediate possession, allowing even low-income individuals to endure the situation. These factors complicated the meaning of income and should be disentangled before unveiling the true nature of how income level associated with depression during the pandemic.

Partly supporting the third hypothesis, individuals who had reduced wages or were involuntarily furloughed from work faced a significantly higher chance of being depressed compared to individuals whose income or employment status was unruffled by COVID-19. More specifically, they were more vulnerable to elevated depressed mood but not diminished positive emotions. Being furloughed could be a burdensome financial stressor that also amplified feelings of uncertainty and helplessness, further exacerbating depression.

\section{Severity}

Severity was not directedly related to depression or depressed mood after controlling for several covariates. However, the severity of COVID-19 exposure was a moderator for the relationship between some demographic correlates and depression as well as depressed mood. First, female participants living in regions of high severity were most susceptible to depression and depressed mood when compared to male 
participants. Hence, in less severely impacted areas, like Shanxi or Sichuan, women and men were more equally affected by the repercussions of COVID-19 than their counterparts living in SeA, where females were at a disadvantage. Second, more years of education was related to decreased depression and depressed mood in mildly impacted areas, but not in severely impacted areas. Education, being a consistent protective factor against the destructive consequences of COVID-19, was more effective when risk remained low and manageable, yet as severity rose, education lost its benefits. The findings highlighted unique challenges that residents in SeA encountered, possibly due to a combination of stressors, including more significant perceived discrimination, stricter lockdown policy, and a higher risk of contracting the virus.

Contrary to the findings of depression and depressed mood, severity was negatively associated with changes in positive emotions. Neither of the six interaction terms yielded significant results. A subsequent simple slope analysis of the marginally significant relationship between education and severity suggested that more years of education was associated with greater positive emotion regardless of severity. However, the effect was stronger in MiA or MoA.

Gender, age, education, marital status, changes in employment status were pertinent factors to consider when studying changes in the psychological well-being of those facing the pandemic. Throughout the investigation, younger age and lower educational attainment were consistent risk factors for depression, whereas, gender, marital status, and being furloughed from work were situation-specific. The pattern of positive emotion was distinct from models of depression and depressed mood, suggesting that positive affect operated through a distinctive pathway to depression. The findings illustrated that outcomes differed depending on symptoms assessed, accentuating the need to identify symptoms of interest and to match them with the most appropriate and applicable scale or measurement approach.

\section{Limitation}

Several limitations of the study should be acknowledged. The first limitation concerns the sampling strategy of the comparison norm. Our goals were to compare a sample collected during a normal situation (i.e., 2016) with one collected during a global emergency, and further examine whether there were differences among participants from severely, moderately, and mildly affected areas (i.e., 2020). Given the nature of a convenient sample in 2020, concerns might arise as to if they truly represented residents of each province investigated. Although we did adopt an adequate size of sample and recruited from multiple platforms to avoid sampling and estimation biases, future studies with both pre- and post-pandemic data in the same sample may better control for potential confounds. Second, the self-reported CES-D scale was only suitable for evaluating levels of depression and not anxiety, since they are highly comorbid, particularly given that $29 \%$ of respondents reported moderate to severe anxiety symptoms in another study (24). CES-D was adopted here because the primary interest was to assess depression. Other studies are encouraged to evaluate a wide range of outcomes. Third, actual stressors specific to COVID-19 and the experience of containment were not assessed and precluded to keep the survey brief. It is unclear how unique stressors, such as contact history with confirmed cases, health status, pre-existing mental disorder, lack of socioeconomic resources, and stigma, are related to psychological distress.

\section{CONCLUSION}

The study examined a narrow range of psychological consequences of COVID-19 in Chinese residents who were living in MiA, MoA, and SeA, compared to a baseline group living in the same provinces in 2016. The outbreak was related to individuals' increased symptoms of depression, elevated levels of depressed mood, and diminished positive emotions. Stigma and local government policies may stir waves of distrust among neighbors and friends and feelings of marginalization and isolation, especially for residents in the Hubei province. Timely psychological interventions are necessary for individuals in need, particularly those who are single, unemployed due to COVID-19, and have fewer years of education.

\section{DATA AVAILABILITY STATEMENT}

The raw data supporting the conclusions of this article will be made available by the authors, without undue reservation.

\section{ETHICS STATEMENT}

The studies involving human participants were reviewed and approved by Institutional Review Board of Tsinghua University, Department of Psychology. The patients/participants provided their written informed consent to participate in this study.

\section{AUTHOR CONTRIBUTIONS}

$\mathrm{XH}, \mathrm{ZY}, \mathrm{KB}$, and SC designed the study and analyzed the data under the supervision of PS. XH, SC, KB, ZY, and PS discussed the results and wrote the paper together. All authors contributed to the article and approved the submitted version.

\section{FUNDING}

This research received funding from the Tsinghua University Spring Breeze Fund (20201080524), the China Narcotics Control Foundation (CNCF-2016-22) and the Natural Science Foundation of China (81671065). 


\section{REFERENCES}

1. Ghebreyesus TA. WHO Director-General's Remarks at the Media Briefing on 2019- $n \mathrm{CoV}$ on 11 February 2020. World Health Organization (2020). Available online at: https://www.who.int/director-general/speeches/detail/ who-director-general-s-remarks-at-the-media-briefing-on-2019-ncov-on11-february-2020 (accessed May 24, 2020).

2. John Hopkins University. COVID-19 Dashboard by the Center for Systems Science and Engineering (CSSE) at Johns Hopkins University (JHU). (2020). Available online at: https://www.arcgis.com/apps/opsdashboard/index.html\#/ bda7594740fd40299423467b48e9ecf6 (accessed July 29, 2020).

3. Gruber J, Prinstein MJ, Clark LA, Rottenberg J, Abramowitz JS, Albano AM, et al. Mental health and clinical psychological science in the time of COVID19: Challenges, opportunities, and a call to action. Am Psychol. (2020). doi: 10.1037/amp0000707. [Epub ahead of print].

4. Asmundson G, Taylor S. Coronaphobia: Fear and the 2019-nCoV outbreak. J Anxiety Disord. (2020) 70:102196. doi: 10.1016/j.janxdis.2020.102196

5. Roberton T, Carter ED, Chou VB, Stegmuller AR, Jackson BD, Tam $\mathrm{Y}$, et al. Early estimates of the indirect effects of the COVID-19 pandemic on maternal and child mortality in low-income and middleincome countries: a modelling study. Lancet Global Health. (2020) 8:e9018. doi: 10.1016/S2214-109X(20)30229-1

6. Bavel JJV, Baicker K, Boggio PS, Capraro V, Cichocka A, Cikara M, et al. Using social and behavioural science to support COVID-19 pandemic response. Nat Human Behav. (2020) 4:460-71. doi: 10.1038/s41562-020-0884-z

7. Schaller M, Neuberg SL. Chapter one-danger, disease, and the nature of prejudice(s). In: Olson JM, Zanna MP, editors. Advances in Experimental Social Psychology. Vol. 46. Academic Press (2012). p. 1-54. doi: 10.1016/B978-0-12-394281-4.00001-5

8. He J, He L, Zhou W, Nie X, He M. Discrimination and social exclusion in the outbreak of COVID-19. Int J Environ Res Public Health. (2020) 17:2933. doi: 10.3390/ijerph17082933

9. Paradies Y, Ben J, Denson N, Elias A, Priest N, Pieterse A, et al. Racism as a determinant of health: A systematic review and meta-analysis. PLoS ONE. (2015) 10:e0138511. doi: 10.1371/journal.pone.0138511

10. Feng E, Cheng A. Restrictions and Rewards: How China is Locking down Half a Billion Citizens. (2020). Available online at: https://www.npr.org/sections/ goatsandsoda/2020/02/21/806958341/restrictions-and-rewards-how-chinais-locking-down-half-a-billion-citizens (accessed May 25, 2020).

11. Baumeister RF, Leary MR. The need to belong: desire for interpersonal attachments as a fundamental human motivation. Psychol Bull. (1995) 117:497-529. doi: 10.1037/0033-2909.117.3.497

12. Brooks SK, Webster RK, Smith LE, Woodland L, Wessely S, Greenberg N, et al. The psychological impact of quarantine and how to reduce it: Rapid review of the evidence. Lancet. (2020) 395:912-20. doi: 10.1016/S0140-6736(20)30460-8

13. Lei L, Huang $\mathrm{X}$, Zhang S, Yang J, Yang L, Xu M. Comparison of prevalence and associated factors of anxiety and depression among people affected by versus people unaffected by quarantine during the COVID-19 epidemic in southwestern china. Med Sci Monitor. (2020) 26:e924609. doi: 10.12659/MSM.924609

14. Donoho CJ, Bonanno GA, Porter B, Kearney L, Powell TM. A decade of war: Prospective trajectories of posttraumatic stress disorder symptoms among deployed us military personnel and the influence of combat exposure. Am Epidemiol J. (2017) 186:1310-8. doi: 10.1093/aje/kwx318

15. Porter B, Hoge CW, Tobin LE, Donoho CJ, Castro CA, Luxton DD, et al. Measuring aggregated and specific combat exposures: Associations between combat exposure measures and posttraumatic stress disorder, depression, alcohol-related problems. J Trauma Stress. (2018) 31:296306. doi: $10.1002 /$ jts. 22273

16. Sinclair VG, Wallston KA, Strachan E. Resilient coping moderates the effect of trauma exposure on depression. Res Nurs Health. (2016) 39:24452. doi: 10.1002/nur.21723

17. Bonanno GA, Brewin CR, Kaniasty $\mathrm{K}$, Greca AML. Weighing the costs of disaster: Consequences, risks, and resilience in individuals, families, and communities. Psychol Sci Public Interest. (2010) 11:149. doi: $10.1177 / 1529100610387086$

18. Warmingham JM, Handley ED, Rogosch FA, Manly JT, Cicchetti D. Identifying maltreatment subgroups with patterns of maltreatment subtype and chronicity: A latent class analysis approach. Const Child Maltreat. (2019) 87:28-39. doi: 10.1016/j.chiabu.2018.08.013

19. De Brier N, Stroobants S, Vandekerckhove P, De Buck E. Factors affecting mental health of health care workers during coronavirus disease outbreaks (SARS, MERS \& COVID-19): a rapid systematic review. PLoS ONE. (2020) 15:e0244052. doi: 10.1371/journal.pone.0244052

20. Lai J, Ma S, Wang $\mathrm{Y}$, Cai $\mathrm{Z}, \mathrm{Hu}$ J, Wei $\mathrm{N}$, et al. Factors associated with mental health outcomes among health care workers exposed to coronavirus disease 2019. JAMA Network Open. (2020) 3:e203976. doi: 10.1001/jamanetworkopen.202 0.3976

21. Wu P, Fang Y, Guan Z, Fan B, Kong J, Yao Z, et al. The psychological impact of the sars epidemic on hospital employees in China: exposure, risk perception, and altruistic acceptance of risk. Canad J Psychiatry. (2009) 54:302-11. doi: 10.1177/070674370905400504

22. Peng $\mathrm{M}$, Mo B, Liu $\mathrm{Y}$, Xu M, Song $\mathrm{X}$, Liu L, et al. Prevalence, risk factors and clinical correlates of depression in quarantined population during the COVID-19 outbreak. J Affect Disord. (2020) 275:119-24. doi: 10.1016/j.jad.2020.06.035F

23. Qiu J, Shen B, Zhao M, Wang Z, Xie B, Xu Y. A nationwide survey of psychological distress among Chinese people in the COVID-19 epidemic: implications and policy recommendations. Gen Psychiatry. (2020) 33:e100213. doi: 10.1136/gpsych-2020-100213

24. Wang C, Pan R, Wan X, Tan Y, Xu L, Ho CS, et al. Immediate psychological responses and associated factors during the initial stage of the 2019 coronavirus disease (COVID-19) epidemic among the general population in China. Int J Environ Res Public Health. (2020) 17:1729. doi: 10.3390/ijerph17051729

25. László KD, Pikhart H, Kopp MS, Bobak M, Pajak A, Malyutina S, et al. Job insecurity and health: a study of 16 European countries. Soc Sci Med. (2010) 70:867-74. doi: 10.1016/j.socscimed.2009.11.022

26. Kim TJ, von dem Knesebeck O. Perceived job insecurity, unemployment and depressive symptoms: a systematic review and meta-analysis of prospective observational studies. Int Arch Occupat Environ Health. (2016) 89:56173. doi: $10.1007 / \mathrm{s} 00420-015-1107-1$

27. Rohde N, Tang KK, Osberg L, Rao P. The effect of economic insecurity on mental health: recent evidence from Australian panel data. Soc Sci Med. (2016) 151:250-8. doi: 10.1016/j.socscimed.2015.12.014

28. Forbes MK, Krueger RF. The great recession and mental health in the United States. Clin Psychol Sci. (2019) 7:90013. doi: $10.1177 / 2167702619859337$

29. Wilson JM, Lee J, Fitzgerald HN, Oosterhoff B, Sevi B, Shook NJ. Job insecurity and financial concern during the COVID-19 pandemic are associated with worse mental health. J Occup Environ Med. (2020) 62:68691. doi: 10.1097/JOM.0000000000001962

30. Eberly LE. Multiple linear regression. In: Ambrosius WT, editor. Topics in Biostatistics. New Jersey: Humana Press (2007). p. 165-87. doi: 10.1007/978-1-59745-530-5_9

31. Slinker BK, Glantz SA. Multiple linear regression. Circulation. (2008) 117:1732-7. doi: 10.1161/CIRCULATIONAHA.106.654376

32. Institute of Social Science Survey PU. China Family Panel Studies (CFPS) (Peking University \& National Natural Science Foundation of China, Trans.; V39 ed.). Peking University Open Research Data Platform (2010). doi: $10.18170 / \mathrm{DVN} / 45 \mathrm{LCSO}$

33. Liu JL, Ren F, Fang YS. The model fit of CES-D in Chinese adult sample. $A d v$ Psychol. (2018) 8:1829-37. doi: 10.12677/ap.2018.812213

34. Zhang J, Wu ZY, Fang G, Li J, Han BX, Chen ZX. Establishing a national urban norm for the center for epidemiology scale for depression. Chin Mental Health J. (2010) 24:139-43. doi: 10.3969/j.issn.1000-6729.2010.02.015

35. Radloff LS. The CES-D scale: a self-report depression scale for research in the general population. Appl Psychol Meas. (1977) 1:385-401. doi: 10.1177/014662167700100306

36. Carleton RN, Thibodeau MA, Teale MJN, Welch PG, Abrams MP, Robinson $\mathrm{T}$, et al. The center for epidemiologic studies depression scale: a review with a theoretical and empirical examination of item content and factor structure. PLoS ONE. (2013) 8:e58067. doi: 10.1371/journal.pone.0058067

37. Edwards MC, Cheavens JS, Heiy JE, Cukrowicz KC. A reexamination of the factor structure of the center for epidemiologic studies depression 
scale: is a one-factor model plausible? Psychol Assess. (2010) 22:7115. doi: 10.1037/a0019917

38. R Core Team. R: A Language and Environment for Statistical Computing. Vienna: R Foundation for Statistical Computing (2013).

39. Aiken LS, West SG. Multiple Regression: Testing and Interpreting Interactions. Newbury Park; Thousand Oaks, CA: Sage Publications, Inc. (1991).

40. Cohen J, Cohen P. Applied Multiple Regression/correlation Analysis for the Behavioral Sciences: Vol. 2nd ed. New York, NY: Psychology Press (1983).

41. Robinson C, Tomek S, Schumacker R. Tests of moderation effects: difference in simple slopes versus the interaction term. Multiple Linear Regress Viewpoints. (2013) 39:16-24.

42. Vilagut G, Forero CG, Barbaglia G, Alonso J. Screening for depression in the general population with the center for epidemiologic studies depression (CES-D): a systematic review with meta-analysis. PLoS ONE. (2016) 11:e0155431. doi: 10.1371/journal.pone.0155431

43. Wilcox RR. Introduction to Robust Estimation and Hypothesis Testing. Burlington, MA: Elsevier Academic Press (2005).

44. Guo J, Feng XL, Wang XH, van IJzendoorn MH. Coping with COVID-19: exposure to COVID-19 and negative impact on livelihood predict elevated mental health problems in Chinese adults. Int J Environ Res Pub Health. (2020) 17:3857. doi: 10.3390/ijerph17113857

45. Iqbal SZ, Li B, Onigu-Otito E, Naqvi MF, Shah AA. The long-term mental health effects of COVID-19. Psychiatr Ann. (2020) 50:5225. doi: 10.3928/00485713-20201103-01

46. Pascoe EA, Smart Richman L. Perceived discrimination and health: a metaanalytic review. Psychol Bull. (2009) 135:531-54. doi: 10.1037/a0016059

47. Zhong B, Huang Y, Liu Q. Mental health toll from the coronavirus: social media usage reveals Wuhan residents' depression and secondary trauma in the COVID-19 outbreak. Comput Human Behav. (2021) 114:106524. doi: 10.1016/j.chb.2020.106524

48. Varma P, Junge M, Meaklim H, Jackson ML. Younger people are more vulnerable to stress, anxiety and depression during COVID-19 pandemic: a global cross-sectional survey. Prog Neuro-Psychopharmacol Biol Psychiatry. (2020) 109:110236. doi: 10.1016/j.pnpbp.2020.110236

49. Hawryluck L, Gold WL, Robinson S, Pogorski S, Galea S, Styra R. SARS control and psychological effects of quarantine, Toronto, Canada. Emerg Infect Dis. (2004) 10:1206-12. doi: 10.3201/eid1007.030703

50. Hwang TJ, Rabheru K, Peisah C, Reichman W, Ikeda M. Loneliness and social isolation during the COVID-19 pandemic. Int Psychogeriatr. (2020) 32:1217-20. doi: 10.1017/S1041610220000988

51. Gómez-Salgado J, Andrés-Villas M, Domínguez-Salas S, Díaz-Milanés D, Ruiz-Frutos C. Related health factors of psychological distress during the COVID-19 pandemic in Spain. Int J Environ Res Public Health. (2020) 17:3947. doi: 10.3390/ijerph17113947

52. Solomou I, Constantinidou F. Prevalence and predictors of anxiety and depression symptoms during the COVID-19 pandemic and compliance with precautionary measures: age and sex matter. Int J Environ Res Public Health. (2020) 17:4924. doi: 10.3390/ijerph17144924

53. Mazza C, Ricci E, Biondi S, Colasanti M, Ferracuti S, Napoli C, et al. A Nationwide survey of psychological distress among Italian people during the COVID-19 pandemic: immediate psychological responses and associated factors. Int J Environ Res Public Health. (2020) 17:3165. doi: 10.3390/ijerph17093165
54. Maji S. Society and good woman: a critical review of gender difference in depression. Int J Soc Psychiatry. (2018) 64:396405. doi: $10.1177 / 0020764018765023$

55. Owen L. Coronavirus: Five Ways Virus Upheaval is Hitting Women in Asia. BBC News. (2020). Available online at: https://www.bbc.com/news/worldasia-51705199 (accessed May 25, 2020).

56. Bjelland I, Krokstad S, Mykletun A, Dahl AA, Tell GS, Tambs K. Does a higher educational level protect against anxiety and depression? The HUNT study. Soc Sci Med. (2008) 66:1334-45. doi: 10.1016/j.socscimed.2007.12.019

57. Chang-Quan H, Zheng-Rong W, Yong-Hong L, Yi-Zhou X, Qing-Xiu L. Education and risk for late life depression: a meta-analysis of published literature. Int J Psychiatry Med. (2010) 40:109-24. doi: 10.2190/PM.40.1.i

58. Rai D, Zitko P, Jones K, Lynch J, Araya R. Country- and individual-level socioeconomic determinants of depression: Multilevel cross-national comparison. Brit J Psychiatry. (2013) 202:195-203. doi: 10.1192/bjp.bp.112.112482

59. Ettman CK, Abdalla SM, Cohen GH, Sampson L, Vivier PM, Galea S. Low assets and financial stressors associated with higher depression during COVID-19 in a nationally representative sample of US adults. J Epidemiol Commun Health. (2020). doi: 10.1136/jech-2020-215213. [Epub ahead of print].

60. Shi L, Lu, ZA, Que JY, Huang L, Liu L, Ran MS, et al. Prevalence of and risk factors associated with mental health symptoms among the general population in China during the coronavirus disease 2019 pandemic. JAMA Network Open. (2020) 3:e2014053. doi: 10.1001/jamanetworkopen.2020.14053

61. Wenning F, Wang C, Zou L, Yingying G, Zuxun L, Shijiao Y, et al. Psychological health, sleep quality, and coping styles to stress facing the COVID-19 in Wuhan, China. Transl Psychiatry. (2020) 10:225. doi: 10.1038/s41398-020-00913-3

62. Liu R, Li T, Greene R. Migration and inequality in rental housing: affordability stress in the Chinese cities. Appl Geography. (2020) 115:102138. doi: 10.1016/j.apgeog.2019.102138

63. Zeng $\mathrm{H}, \mathrm{Yu} \mathrm{X}$, Zhang J. Urban village demolition, migrant workers' rental costs and housing choices: Evidence from Hangzhou, China. Cities. (2019) 94:70-9. doi: 10.1016/j.cities.2019.05.029

64. Chentsova Dutton YE, Choi, IJ, Choi E. Perceived parental support and adolescents' positive self-beliefs and levels of distress across four countries. Front Psychol. (2020) 11:353. doi: 10.3389/fpsyg.2020. 00353

65. Shoham A, Malul M. The role of cultural attributes in savings rates. Cross Cult Manage. (2012) 19:304-14. doi: 10.1108/13527601211247062

Conflict of Interest: The authors declare that the research was conducted in the absence of any commercial or financial relationships that could be construed as a potential conflict of interest.

Copyright $\odot 2021$ Han, Chen, Bi, Yang and Sun. This is an open-access article distributed under the terms of the Creative Commons Attribution License (CC BY). The use, distribution or reproduction in other forums is permitted, provided the original author(s) and the copyright owner(s) are credited and that the original publication in this journal is cited, in accordance with accepted academic practice. No use, distribution or reproduction is permitted which does not comply with these terms. 